\title{
Dedekind cotangent sums
}

\author{
by \\ Matthias Beck (Binghamton, NY)
}

1. Introduction. While studying the transformation properties of

$$
\eta(z):=e^{\pi i z / 12} \prod_{n \geq 1}\left(1-e^{2 \pi i n z}\right),
$$

under $\mathrm{SL}_{2}(\mathbb{Z})$, Dedekind, in the 1880's [De], naturally arrived at the following arithmetic function: Let $((x))$ be the sawtooth function defined by

$$
((x)):= \begin{cases}\{x\}-1 / 2 & \text { if } x \notin \mathbb{Z}, \\ 0 & \text { if } x \in \mathbb{Z} .\end{cases}
$$

Here $\{x\}=x-[x]$ denotes the fractional part of $x$. For $a, b \in \mathbb{N}:=\{n \in \mathbb{Z}$ : $n>0\}$, we define the Dedekind sum as

$$
\mathfrak{s}(a, b):=\sum_{k \bmod b}\left(\left(\frac{k a}{b}\right)\right)\left(\left(\frac{k}{b}\right)\right) .
$$

The Dedekind sums and their generalizations have since intrigued mathematicians from various areas such as analytic [De, Di1, Al] and algebraic number theory [Me, S], topology [HZ, Za, MS], algebraic [P, BV] and combinatorial geometry [Mo, DR], and algorithmic complexity [Knu].

By means of the discrete Fourier series of the sawtooth function (see, for example, [RG, p. 14]),

$$
\left(\left(\frac{n}{p}\right)\right)=\frac{i}{2 p} \sum_{k=1}^{p-1} \cot \left(\frac{\pi k}{p}\right) e^{2 \pi i k n / p}
$$

it is not hard to write the Dedekind sum in terms of cotangents:

$$
\mathfrak{s}(a, b)=\frac{1}{4 b} \sum_{k=1}^{b-1} \cot \frac{\pi k a}{b} \cot \frac{\pi k}{b} .
$$

2000 Mathematics Subject Classification: Primary 11L03; Secondary 52C07.

Key words and phrases: Dedekind sum, cotangent sum, Petersson-Knopp identity, lattice point enumeration in polyhedra. 
Starting with these two representations (2) and (4) of $\mathfrak{s}(a, b)$, various generalizations of the Dedekind sum have been introduced. This paper constitutes an attempt to unify generalizations of the Dedekind sum in its "cotangent representation" (4). Through the discrete Fourier series (3), this includes most generalizations of the "sawtooth representation" (2) of the Dedekind sum.

Let $\cot ^{(m)}$ denote the $m$ th derivative of the cotangent function. Our generalized Dedekind sum is introduced as follows:

Definition 1. For $a_{0}, \ldots, a_{d} \in \mathbb{N}, m_{0}, \ldots, m_{d} \in \mathbb{N}_{0}:=\mathbb{N} \cup\{0\}$, $z_{0}, \ldots, z_{d} \in \mathbb{C}$, we define the Dedekind cotangent sum as

$$
\mathfrak{c}\left(\begin{array}{c|ccc}
a_{0} & a_{1} & \ldots & a_{d} \\
m_{0} & m_{1} & \ldots & m_{d} \\
z_{0} & z_{1} & \ldots & z_{d}
\end{array}\right):=\frac{1}{a_{0}^{m_{0}+1}} \sum_{k \bmod a_{0}} \prod_{j=1}^{d} \cot ^{\left(m_{j}\right)} \pi\left(a_{j} \frac{k+z_{0}}{a_{0}}-z_{j}\right),
$$

where the sum is taken over all $k \bmod a_{0}$ for which the summand is not singular.

The Dedekind cotangent sums include as special cases various generalized Dedekind sums introduced by Rademacher [R], Apostol [Ap], Carlitz [C1], Zagier [Za], Berndt [Be], Meyer, Sczech [MS], and Dieter [Di2]. Section 2 contains the definitions of these previously defined sums and the connections to our new definition.

The most fundamental and important theorems for any of the generalized Dedekind sums are the reciprocity laws: an appropriate sum of generalized Dedekind sums (usually permuting the arguments in a cyclic fashion) gives a simple rational expression. The famous reciprocity law for the classical Dedekind sum is as old as the sum itself:

TheOREM 1 (Dedekind). If $a, b \in \mathbb{N}$ are relatively prime then

$$
\mathfrak{s}(a, b)+\mathfrak{s}(b, a)=-\frac{1}{4}+\frac{1}{12}\left(\frac{a}{b}+\frac{1}{a b}+\frac{b}{a}\right) .
$$

The following reciprocity law for the Dedekind cotangent sums will be proved in Section 3:

Theorem 2. Let $a_{0}, \ldots, a_{d} \in \mathbb{N}, m_{0}, \ldots, m_{d} \in \mathbb{N}_{0}, z_{0}, \ldots, z_{d} \in \mathbb{C}$. If for all distinct $i, j \in\{0, \ldots, d\}$ and all $m, n \in \mathbb{Z}$,

$$
\frac{m+z_{i}}{a_{i}}-\frac{n+z_{j}}{a_{j}} \notin \mathbb{Z}
$$

then

$$
\sum_{n=0}^{d}(-1)^{m_{n}} m_{n} ! \sum_{\substack{l_{0}, \ldots, \hat{l_{n}}, \ldots, l_{d} \geq 0 \\ l_{0}+\ldots+\hat{l_{n}}+\ldots+l_{d}=m_{n}}} \frac{a_{0}^{l_{0}} \ldots \widehat{a_{n}^{l_{n}}} \ldots a_{d}^{l_{d}}}{l_{0} ! \ldots \widehat{l_{n} !} \ldots l_{d} !}
$$




$$
\begin{aligned}
& \times \mathfrak{c}\left(\begin{array}{c|ccccc}
a_{n} & a_{0} & \ldots & \widehat{a_{n}} & \ldots & a_{d} \\
m_{n} & m_{0}+l_{0} & \ldots & m_{n}+l_{n} & \ldots & m_{d}+l_{d} \\
z_{n} & z_{0} & \ldots & \widehat{z_{n}} & \ldots & z_{d}
\end{array}\right) \\
& =\left\{\begin{array}{lll}
(-1)^{d / 2} & \text { if all } m_{k}=0 & \text { and } d \text { is even, } \\
0 & \text { otherwise. }
\end{array}\right.
\end{aligned}
$$

As usual, $\widehat{x_{n}}$ means we omit the term $x_{n}$.

In Section 4, we obtain as corollaries the reciprocity laws for Dedekind's, Apostol's, Meyer-Sczech's, Dieter's and Zagier's generalized Dedekind sums. The purpose here is not so much a rederivation of old theorems but rather to show a common thread to all of them.

Another basic identity on the classical Dedekind sum is about a hundred years younger than Dedekind's reciprocity law [Kno]:

Theorem 3 (Petersson-Knopp). Suppose $a, b \in \mathbb{N}$ are relatively prime. Then

$$
\sum_{d \mid n} \sum_{k \bmod d} \mathfrak{s}\left(\frac{n}{d} b+k a, a d\right)=\sigma(n) \mathfrak{s}(b, a) .
$$

Here $\sigma(n)$ denotes the sum of the positive divisors of $n$.

This result has also been extended to certain generalized Dedekind sums $[\mathrm{AV}, \mathrm{PR}, \mathrm{Zh}]$. The respective identity for the cotangent sum follows from a much more general theorem, stated and proved in Section 5:

THEOREM 4. For $n, a_{0}, \ldots, a_{d} \in \mathbb{N}, m_{0}, \ldots, m_{d} \in \mathbb{N}_{0}$,

$$
\begin{gathered}
\sum_{b \mid n} b^{m_{0}+1-m_{1}-\ldots-m_{d}-d} \\
\times \sum_{r_{1}, \ldots, r_{d} \bmod b} \mathfrak{c}\left(\begin{array}{c|cccc}
a_{0} b & \frac{n}{b} a_{1}+r_{1} a_{0} & \ldots & \frac{n}{b} a_{d}+r_{d} a_{0} \\
m_{0} & m_{1} & \ldots & m_{d} \\
0 & 0 & \ldots & 0
\end{array}\right) \\
=n \sigma_{-m_{1}-\ldots-m_{d}-1}(n) \mathfrak{c}\left(\begin{array}{c|c|ccc}
a_{0} & a_{1} & \ldots & a_{d} \\
m_{0} & m_{1} & \ldots & m_{d} \\
0 & 0 & \ldots & 0
\end{array}\right) .
\end{gathered}
$$

Here $\sigma_{m}(n):=\sum_{d \mid n} d^{m}$.

Finally, we show in Section 6 a computability result:

THEOREM 5. The Dedekind cotangent sum

$$
\mathfrak{c}\left(\begin{array}{c|ccc}
a_{0} & a_{1} & \ldots & a_{d} \\
m_{0} & m_{1} & \ldots & m_{d} \\
z_{0} & z_{1} & \ldots & z_{d}
\end{array}\right)
$$

is polynomial-time computable in the input size of $a_{0}, \ldots, a_{d}$. 
Computability for any of the generalized Dedekind sums involving two integer arguments follows easily from the two-term reciprocity laws. However, the reciprocity laws for "higher-dimensional" analogues do not imply nice computability properties.

2. Various Dedekind sums. In this section we will give an overview of previously defined generalizations of the Dedekind sum. We do not claim any completeness but hope to give a good picture of what has been introduced in the past.

The sawtooth function $((x))$ defined in (1) is the first Bernoulli function $\bar{B}_{1}(x)$, defined to be zero at the integers. We used the slightly antiquated function $((x))$ partly for historical reasons, partly because it has a simpler discrete Fourier series. The Bernoulli polynomials $B_{k}(x)$ are defined through

$$
\frac{z e^{x z}}{e^{z}-1}=\sum_{k \geq 0} \frac{B_{k}(x)}{k !} z^{k}
$$

The first few of them are

$$
\begin{array}{llrl}
B_{1}(x) & =x-\frac{1}{2}, & B_{5}(x) & =x^{5}-\frac{5}{2} x^{4}+\frac{5}{3} x^{3}-\frac{1}{6} x, \\
B_{2}(x) & =x^{2}-x+\frac{1}{6}, & B_{6}(x) & =x^{6}-3 x^{5}+\frac{5}{2} x^{4}+\frac{1}{2} x^{2}+\frac{1}{42}, \\
B_{3}(x) & =x^{3}-\frac{3}{2} x^{2}+\frac{1}{2} x, & B_{7}(x) & =x^{7}-\frac{7}{2} x^{6}+\frac{7}{2} x^{5}+\frac{7}{6} x^{3}+\frac{1}{6} x . \\
B_{4}(x) & =x^{4}-2 x^{3}+x^{2}-\frac{1}{30}, & &
\end{array}
$$

The Bernoulli numbers are $B_{k}:=B_{k}(0)$. The Bernoulli functions $\bar{B}_{k}(x)$ are the periodized Bernoulli polynomials:

$$
\bar{B}_{k}(x):=B_{k}(\{x\}) .
$$

Apostol [Ap] replaced one of the sawtooth functions in (2) by an arbitrary Bernoulli function:

$$
\sum_{k \bmod b} \frac{k}{b} \bar{B}_{n}\left(\frac{k a}{b}\right)
$$

Apostol's idea was generalized by Carlitz [C1] and Mikolás [Mi] to what we would like to call the Dedekind-Bernoulli sum, defined for $a, b, c, m, n \in \mathbb{N}$ as

$$
\mathfrak{s}_{m, n}(a ; b, c):=\sum_{k \bmod b} \bar{B}_{m}\left(\frac{k b}{a}\right) \bar{B}_{n}\left(\frac{k c}{a}\right) .
$$

Another way of generalizing (2) is to shift the argument of the sawtooth functions. This was introduced by Meyer [Me] and Dieter [Di1], and brought to a solid ground by Rademacher $[\mathrm{R}]$ : For $a, b \in \mathbb{N}, x, y \in \mathbb{R}$, the Dedekind- 
Rademacher sum is defined by

$$
\mathfrak{s}(a, b ; x, y):=\sum_{k \bmod b}\left(\left(a \frac{k+y}{b}-x\right)\right)\left(\left(\frac{k+y}{b}\right)\right) .
$$

Note that there is no loss in restricting $x$ and $y$ to $0 \leq x, y<1$.

The ideas of Apostol and Rademacher can also be combined: Takács [T] introduced a shift in Apostol's sum (7):

$$
\sum_{k \bmod b} \bar{B}_{1}\left(\frac{k+y}{b}\right) \bar{B}_{n}\left(a \frac{k+y}{b}-x\right) \text {. }
$$

This was further generalized by Halbritter $[\mathrm{H}]$ and later by Hall, Wilson, and Zagier [HWZ] to the generalized Dedekind-Rademacher sum, defined for $a, b, c, m, n \in \mathbb{N}, x, y, z \in \mathbb{R}$ by

$$
\mathfrak{s}_{m, n}\left(\begin{array}{l|ll}
a & b & c \\
x & y & z
\end{array}\right):=\sum_{k \bmod a} \bar{B}_{m}\left(b \frac{k+x}{a}-y\right) \bar{B}_{n}\left(c \frac{k+x}{a}-z\right) .
$$

On the other hand, we can start with the cotangent representation (4) of the Dedekind sum to arrive at generalizations. The equivalent of the DedekindRademacher sum in terms of cotangents was first defined by Meyer and Sczech [MS], motivated by the appearance of the classical Dedekind sum in topology [HZ]. The version we state here was introduced by Dieter [Di2]: For $a, b, c \in \mathbb{N}, x, y, z \in \mathbb{R}$, the cotangent sum is defined by

$$
\mathfrak{c}(a, b, c ; x, y, z):=\frac{1}{c} \sum_{k \bmod c} \cot \pi\left(a \frac{k+z}{c}-x\right) \cot \pi\left(b \frac{k+z}{c}-y\right) .
$$

Here the sum is taken over all $k \bmod b$ for which the summand is not singular. Dieter remarked in [Di2] that the cotangent sums include as special cases various modified Dedekind sums introduced by Berndt [Be, BD]. Most of them are inspired by the transformation properties of the logarithm of the classical theta-function. We list them here using Berndt's notation; throughout, $a$ and $b$ denote relatively prime positive integers. In the first sum, $\alpha, \beta \in \mathbb{N}$, and $a^{-1}$ is defined through $a^{-1} a \equiv 1 \bmod b$.

$$
\begin{aligned}
s_{\alpha, \beta}(a, b) & :=\sum_{k=1}^{a b-1} \exp \left(2 \pi i\left(\frac{k \alpha}{a}+\frac{k \beta}{b}\right)\right)\left(\left(\frac{k}{a b}\right)\right)\left(\left(\frac{k a^{-1}}{b}\right)\right), \\
S(a, b) & :=\sum_{k=1}^{b-1}(-1)^{k+1+[a k / b]} \\
s_{1}(a, b) & :=\sum_{k=1}^{b}(-1)^{[a k / b]}\left(\left(\frac{k}{b}\right)\right),
\end{aligned}
$$




$$
\begin{aligned}
& s_{2}(a, b):=\sum_{k=1}^{b}(-1)^{k}\left(\left(\frac{k}{b}\right)\right)\left(\left(\frac{k a}{b}\right)\right), \\
& s_{3}(a, b):=\sum_{k=1}^{b}(-1)^{k}\left(\left(\frac{k a}{b}\right)\right), \\
& s_{4}(a, b):=\sum_{k=1}^{b-1}(-1)^{[a k / b]} \\
& s_{5}(a, b):=\sum_{k=1}^{b}(-1)^{k+[a k / b]}\left(\left(\frac{k}{b}\right)\right) .
\end{aligned}
$$

Yet another generalization of (4) we mention here is due to Zagier [Za]. Generalizing the topological considerations in [HZ] to arbitrary dimensions, he arrived naturally at the following expression: Let $a_{1}, \ldots, a_{d} \in \mathbb{N}$ be relatively prime to $a_{0} \in \mathbb{N}$. Define the higher-dimensional Dedekind sum as

$$
\mathfrak{s}\left(a_{0} ; a_{1}, \ldots, a_{d}\right):=\frac{(-1)^{d / 2}}{a_{0}} \sum_{k=1}^{a_{0}-1} \cot \frac{\pi k a_{1}}{a_{0}} \ldots \cot \frac{\pi k a_{d}}{a_{0}} .
$$

We note that this sum vanishes if $d$ is odd, since the cotangent is an odd function. Berndt noticed in $[\mathrm{Be}]$ that a version of the higher-dimensional Dedekind sums was already introduced by Carlitz [C2] via sawtooth functions.

Our Dedekind cotangent sums (Definition 1) combine the cotangent and higher-dimensional Dedekind sums. This could have been done by only introducing a shift of the variable in each cotangent of the higher-dimensional Dedekind sum. The reason for additionally introducing cotangent derivatives is twofold: first, they appear in lattice point enumeration formulas for polyhedra [DR]. We will make use of this fact in Section 6 about the computability of the Dedekind cotangent sums. Second, the cotangent derivatives are essentially the discrete Fourier transforms of the Bernoulli functions. That is, our definition can be seen as the higher-dimensional "cotangent equivalent" to Apostol's Dedekind-Bernoulli sum. In fact, the Dedekind cotangent sums include as special cases all generalized Dedekind sums mentioned in this section with the exception of the Dedekind-Rademacher sum and its generalization by Hall, Wilson, and Zagier. Technically, these sums could be treated in the same manner. What makes a subtle difference is the shift by a real number in the argument of the Bernoulli functions: to handle these, we would have to work with the discrete Fourier series of the shifted Bernoulli functions, which turn out to be much less practical than the ones without a shift.

To be more precise, we state the discrete Fourier series of the Bernoulli functions next. In analogy to (3), we have the straightforward 
Lemma 6. For $m \geq 2$,

$$
\bar{B}_{m}\left(\frac{n}{p}\right)=\frac{B_{m}}{(-p)^{m}}+m\left(\frac{i}{2 p}\right)^{m} \sum_{k=1}^{p-1} \cot ^{(m-1)}\left(\frac{\pi k}{p}\right) e^{2 \pi k n / p}
$$

These discrete Fourier expansions can be used, for example, to rewrite the Dedekind-Bernoulli sums in terms of the Dedekind cotangent sums:

Corollary 7. If $a, b, c \in \mathbb{N}$ are pairwise relatively prime and $m, n \geq 2$ are integers with the same parity then

$$
\begin{aligned}
\mathfrak{s}_{m, n}(a ; b, c) & :=\sum_{k \bmod a} \bar{B}_{m}\left(\frac{k b}{a}\right) \bar{B}_{n}\left(\frac{k c}{a}\right) \\
& =m n \frac{(-1)^{(m-n) / 2}}{2^{m+n} a^{m+n-1}} \sum_{k=1}^{a-1} \cot ^{(m-1)}\left(\frac{\pi k c}{a}\right) \cot ^{(n-1)}\left(\frac{\pi k b}{a}\right)+\frac{B_{m} B_{n}}{a^{m+n-1}} \\
& =: m n \frac{(-1)^{(m-n) / 2}}{2^{m+n}} \mathfrak{c}\left(\begin{array}{c|cc}
m+n-2 & \left.\begin{array}{ccc}
a & c \\
0 & m-1 \\
0 & 0
\end{array}\right)+\frac{B_{m} B_{n}}{a^{m+n-1}} .
\end{array}\right.
\end{aligned}
$$

We note that the parity assumption on $m$ and $n$ is no restriction, since the sums vanish if $m+n$ is odd. It is worth mentioning that a close relative of these sums, namely,

$$
\sum_{k=1}^{a-1} \bar{B}_{m}\left(\frac{k}{a}\right)\left(\left(\frac{k b}{a}\right)\right)=m \frac{(-1)^{(m-1) / 2}}{2^{m+1} a^{m}} \sum_{k=1}^{a-1} \cot \left(\frac{\pi k}{a}\right) \cot ^{(m-1)}\left(\frac{\pi k b}{a}\right)
$$

appears naturally in the study of plane partition enumeration [Al].

Proof of Corollary 7. By Lemma 6,

$$
\begin{aligned}
& \mathfrak{s}_{m, n}(a ; b, c)=\sum_{k \bmod a} \bar{B}_{m}\left(\frac{k b}{a}\right) \bar{B}_{n}\left(\frac{k c}{a}\right) \\
& =m n\left(\frac{i}{2 a}\right)^{m+n} \sum_{k \bmod a} \sum_{j, l=1}^{a-1} \cot ^{(m-1)}\left(\frac{\pi j}{a}\right) \cot ^{(n-1)}\left(\frac{\pi l}{a}\right) e^{2 \pi i k(j b+l c) / a} \\
& +m\left(\frac{i}{2 a}\right)^{m} \frac{B_{n}}{(-a)^{n}} \sum_{k \bmod a} \sum_{j=1}^{a-1} \cot ^{(m-1)}\left(\frac{\pi j}{a}\right) e^{2 \pi i k j b / a} \\
& +n\left(\frac{i}{2 a}\right)^{n} \frac{B_{m}}{(-a)^{m}} \sum_{k \bmod a} \sum_{l=1}^{a-1} \cot ^{(n-1)}\left(\frac{\pi l}{a}\right) e^{2 \pi i k l c / a}+a \frac{B_{m} B_{n}}{(-a)^{m+n}} .
\end{aligned}
$$

Now we use the fact that $m+n$ is even, $a, b, c$ are pairwise relatively prime, 
and

$$
\sum_{k \bmod a} e^{2 \pi i n k / a}= \begin{cases}a & \text { if } a \mid n, \\ 0 & \text { else. }\end{cases}
$$

Hence the first sum in (8) vanishes unless $j b+l c$ is divisible by $a$, that is, $l \equiv-j b c^{-1} \bmod a$, where $c^{-1} c \equiv 1 \bmod a$. The second and third sums in (8) disappear completely, and we are left with

$$
\begin{aligned}
& \mathfrak{s}_{m, n}(a ; b, c) \\
& =m n \frac{(-1)^{(m+n) / 2}}{(2 a)^{m+n}} a \sum_{j=1}^{a-1} \cot ^{(m-1)}\left(\frac{\pi j}{a}\right) \cot ^{(n-1)}\left(-\frac{\pi j b c^{-1}}{a}\right)+\frac{B_{m} B_{n}}{a^{m+n-1}} \\
& =m n \frac{(-1)^{(m+n) / 2}}{2^{m+n} a^{m+n-1}}(-1)^{n} \sum_{j=1}^{a-1} \cot ^{(m-1)}\left(\frac{\pi j c}{a}\right) \cot ^{(n-1)}\left(\frac{\pi j b}{a}\right)+\frac{B_{m} B_{n}}{a^{m+n-1}} .
\end{aligned}
$$

\section{Proof of the reciprocity law}

Proof of Theorem 2. Consider the function

$$
f(z)=\prod_{j=0}^{d} \cot ^{\left(m_{j}\right)} \pi\left(a_{j} z-z_{j}\right) .
$$

We integrate $f$ along the simple rectangular path

$$
\gamma=[x+i y, x-i y, x+1-i y, x+1+i y, x+i y],
$$

where $x$ and $y$ are chosen such that $\gamma$ does not pass through any pole of $f$, and all poles $z_{p}$ of $f$ have imaginary part $\left|\operatorname{Im}\left(z_{p}\right)\right|<y$. By the periodicity of the cotangent, the contributions of the two vertical segments of $\gamma$ cancel each other. By definition of the cotangent,

$$
\lim _{y \rightarrow \infty} \cot (x \pm i y)=\mp i,
$$

and therefore also

$$
\lim _{y \rightarrow \infty} \cot ^{(m)}(x \pm i y)=0
$$

for $m>0$. Hence if any of the $m_{j}>0$, then

$$
\int_{\gamma} f(z) d z=0 .
$$

If all $m_{j}=0$, we obtain

$$
\int_{\gamma} f(z) d z=i^{d+1}-(-i)^{d+1}=i^{d+1}\left(1+(-1)^{d}\right) .
$$


This can be summarized in

$$
\frac{1}{2 i} \int_{\gamma} f(z) d z= \begin{cases}i^{d} & \text { if all } m_{j}=0 \text { and } d \text { is even } \\ 0 & \text { otherwise }\end{cases}
$$

or, by means of the residue theorem,

$$
\pi \sum_{z_{p}} \operatorname{Res}\left(f(z), z_{p}\right)= \begin{cases}i^{d} & \text { if all } m_{j}=0 \text { and } d \text { is even } \\ 0 & \text { otherwise. }\end{cases}
$$

Here the sum ranges over all poles $z_{p}$ inside $\gamma$. It remains to compute their residues. By assumption, $f$ has only simple poles. We will compute the residue at $z_{p}=\left(k+z_{0}\right) / a_{0}, k \in \mathbb{Z}$, the other residues are completely equivalent. We use the Laurent expansion of the cotangent:

$$
\cot \pi\left(a_{0} z-z_{0}\right)=\frac{1}{\pi a_{0}}\left(z-\frac{k+z_{0}}{a_{0}}\right)^{-1}+\text { analytic part }
$$

and, more generally,

$$
\cot ^{\left(m_{0}\right)} \pi\left(a_{0} z-z_{0}\right)=\frac{(-1)^{m_{0}} m_{0} !}{\left(\pi a_{0}\right)^{m_{0}+1}}\left(z-\frac{k+z_{0}}{a_{0}}\right)^{-\left(m_{0}+1\right)}+\text { analytic part. }
$$

The other cotangents are analytic at this pole: for $j>0$,

$$
\begin{aligned}
\cot ^{\left(m_{j}\right)} & \pi\left(a_{j} z-z_{j}\right) \\
= & \sum_{l_{j} \geq 0} \frac{\left(\pi a_{j}\right)^{l_{j}}}{l_{j} !} \cot ^{\left(m_{j}+l_{j}\right)} \pi\left(a_{j} \frac{k+z_{0}}{a_{0}}-z_{j}\right)\left(z-\frac{k+z_{0}}{a_{0}}\right)^{l_{j}} .
\end{aligned}
$$

Hence

$$
\begin{aligned}
& \operatorname{Res}\left(f(z), z=\frac{k+z_{0}}{a_{0}}\right) \\
& \quad=\frac{(-1)^{m_{0}} m_{0} !}{\pi a_{0}^{m_{0}+1}} \sum_{\substack{l_{1}, \ldots, l_{d} \geq 0 \\
l_{1}+\ldots+l_{d}=m_{0}}} \prod_{j=1}^{d} \frac{a_{j}^{l_{j}}}{l_{j} !} \cot ^{\left(m_{j}+l_{j}\right)} \pi\left(a_{j} \frac{k+z_{0}}{a_{0}}-z_{j}\right) .
\end{aligned}
$$

Since $\gamma$ has horizontal width 1 , we have $a_{0}$ poles of the form $\left(k+z_{0}\right) / a_{0}$ inside $\gamma$, where $k$ runs through a complete set of residues modulo $a_{0}$. This gives, by definition of the Dedekind cotangent sum,

$$
\begin{aligned}
& \sum_{k \bmod a_{0}} \operatorname{Res}\left(f(z), z=\frac{k+z_{0}}{a_{0}}\right) \\
& \quad=\frac{1}{\pi}(-1)^{m_{0}} m_{0} ! \sum_{\substack{l_{1}, \ldots, l_{d} \geq 0 \\
l_{1}+\ldots+l_{d}=m_{0}}} \frac{a_{1}^{l_{1}} \ldots a_{d}^{l_{d}}}{l_{1} ! \ldots l_{d} !} \mathfrak{c}\left(\begin{array}{c|ccc}
a_{0} & a_{1} & \ldots & a_{d} \\
m_{0} & m_{1}+l_{1} & \ldots & m_{d}+l_{d} \\
z_{0} & z_{1} & \ldots & z_{d}
\end{array}\right) .
\end{aligned}
$$


The other residues are computed in the same way, and together with (9) give the statement.

4. Variations on a theme. The conditions on the parameters appearing in Theorem 2 are not crucial; however, without them the theorem would not be as easy to state. Indeed, the conditions simply ensure that all poles of the function $f$ used in the proof are simple. We will now state some special cases of Theorem 2 in which we drop some of the conditions. Strictly speaking, these "corollaries" are really corollaries of the proof of Theorem 2. The first of those cases is the classical Dedekind reciprocity law ([De, Theorem 1]). Recall that

$$
\mathfrak{s}(a, b)=\frac{1}{4} \mathfrak{c}\left(\begin{array}{l|ll}
b & a & 1 \\
0 & 0 & 0 \\
0 & 0 & 0
\end{array}\right) .
$$

Proof of Theorem 1. To modify our proof of Theorem 2, we have to consider the function

$$
f(z)=\cot (\pi a z) \cot (\pi b z) \cot (\pi z) .
$$

The residues are computed as above and yield the classical Dedekind sums; the only difference is an additional pole of order three at $z=0$ (we may choose our integration path $\gamma$ such that 0 is inside $\gamma$ ). Its residue is easily computed as

$$
\operatorname{Res}(f(z), z=0)=-\frac{1}{3}\left(\frac{a}{b}+\frac{1}{a b}+\frac{b}{a}\right) .
$$

From here we can proceed as before.

It should be mentioned that a proof of Dedekind's reciprocity law along these lines is given in [RG, p. 21]. In fact, it was this proof that motivated the proof of Theorem 2.

The second special case is an identity equivalent to the reciprocity law for the Dedekind-Bernoulli sums [Ap, C1, Mi]. The respective statement for them can be obtained through Corollary 7 .

Corollary 8. Let $a_{0}, a_{1}, a_{2} \in \mathbb{N}$ be pairwise relatively prime, and $m_{0}, m_{1}, m_{2} \in \mathbb{N}_{0}$ not all zero, such that $m_{0}+m_{1}+m_{2}$ is even. Then

$$
\begin{aligned}
&(-1)^{m_{0}} m_{0} ! \sum_{\substack{l_{1}, l_{2} \geq 0 \\
l_{1}+l_{2}=m_{0}}} \frac{a_{1}^{l_{1}} a_{2}^{l_{2}}}{l_{1} ! l_{2} !} \mathfrak{c}\left(\begin{array}{c|ccc}
a_{0} & a_{1} & a_{2} \\
m_{0} & m_{1}+l_{1} & m_{2}+l_{2} \\
0 & 0 & 0
\end{array}\right) \\
&+(-1)^{m_{1}} m_{1} ! \sum_{\substack{l_{0}, l_{2} \geq 0 \\
l_{0}+l_{2}=m_{1}}} \frac{a_{0}^{l_{0}} a_{2}^{l_{2}}}{l_{0} ! l_{2} !} \mathfrak{c}\left(\begin{array}{c|cc}
a_{1} & a_{0} & a_{2} \\
m_{1} & m_{0}+l_{0} & m_{2}+l_{2} \\
0 & 0 & 0
\end{array}\right)
\end{aligned}
$$




$$
\begin{aligned}
& +(-1)^{m_{2}} m_{2} ! \sum_{\substack{l_{0}, l_{1} \geq 0 \\
l_{0}+l_{1}=m_{2}}} \frac{a_{0}^{l_{0}} a_{1}^{l_{1}}}{l_{0} ! l_{1} !} \mathfrak{c}\left(\begin{array}{c|cc}
a_{2} & a_{0} & a_{1} \\
m_{2} & m_{0}+l_{0} & m_{1}+l_{1} \\
0 & 0 & 0
\end{array}\right) \\
& =\phi\left(a_{0}, a_{1}, a_{2}, m_{0}, m_{1}, m_{2}\right),
\end{aligned}
$$

where

$$
\begin{aligned}
& \phi\left(a_{0}, a_{1}, a_{2}, m_{0}, m_{1}, m_{2}\right):=(-4)^{\left(m_{0}+m_{1}+m_{2}\right) / 2} \\
& \begin{array}{c}
\times\left(\frac{(-1)^{m_{0}}}{a_{0}^{m_{0}+1}} \sum_{\substack{2 k_{1} \geq m_{1}+1 \\
2 k_{2} \geq m_{2}+1 \\
2\left(k_{1}+k_{2}-1\right) \\
=}}\left(\begin{array}{c}
m_{0} \\
2 k_{1}-1-m_{1}
\end{array}\right) \frac{B_{2 k_{1}} B_{2 k_{2}}}{k_{1} k_{2}} a_{1}^{2 k_{1}-1-m_{1}} a_{2}^{2 k_{2}-1-m_{2}}\right. \\
=m_{0}+m_{1}+m_{2}
\end{array} \\
& +\frac{(-1)^{m_{1}}}{a_{1}^{m_{1}+1}} \sum_{\substack{2 k_{0} \geq m_{0}+1 \\
2 k_{2} \geq m_{2}+1 \\
2\left(k_{0}+k_{2}-1\right) \\
=}}\left(\begin{array}{c}
m_{1} \\
2 k_{2}-1-m_{2}
\end{array}\right) \frac{B_{2 k_{0}} B_{2 k_{2}}}{k_{0} k_{2}} a_{0}^{2 k_{0}-1-m_{1}+m_{2}} a_{2}^{2 k_{2}-1-m_{2}} \\
& \begin{array}{c}
\left.+\frac{(-1)^{m_{2}}}{a_{2}^{m_{2}+1}} \sum_{\substack{2 k_{0} \geq m_{0}+1 \\
2 k_{1} \geq m_{1}+1 \\
2\left(k_{0}+k_{1}-1\right) \\
=}}\left(\begin{array}{c}
m_{2} \\
2 k_{0}-1-m_{0}
\end{array}\right) \frac{B_{2 k_{0}} B_{2 k_{1}}}{k_{0} k_{1}} a_{0}^{2 k_{0}-1-m_{0}} a_{1}^{2 k_{1}-1-m_{1}}\right) \\
=m_{0}+m_{1}+m_{2}
\end{array} \\
& +\frac{2^{m_{0}+m_{1}+m_{2}+2} B_{m_{0}+m_{1}+m_{2}+2}}{m_{0}+m_{1}+m_{2}+2}\left(\frac{(-1)^{\left(m_{0}+m_{1}-m_{2}\right) / 2} m_{0} ! m_{1} ! a_{2}^{m_{0}+m_{1}+1}}{\left(m_{0}+m_{1}+1\right) ! a_{0}^{m_{0}+1} a_{1}^{m_{1}+1}}\right. \\
& +\frac{(-1)^{\left(m_{0}+m_{2}-m_{1}\right) / 2} m_{0} ! m_{2} ! a_{1}^{m_{0}+m_{2}+1}}{\left(m_{0}+m_{2}+1\right) ! a_{0}^{m_{0}+1} a_{2}^{m_{2}+1}} \\
& \left.+\frac{(-1)^{\left(m_{1}+m_{2}-m_{0}\right) / 2} m_{1} ! m_{2} ! a_{0}^{m_{1}+m_{2}+1}}{\left(m_{1}+m_{2}+1\right) ! a_{1}^{m_{1}+1} a_{2}^{m_{2}+1}}\right) .
\end{aligned}
$$

Again the parity restriction on $m_{0}+m_{1}+m_{2}$ is no constraint, since otherwise all the sums vanish.

Proof. As in the last proof, we use the function

$$
f(z)=\cot ^{\left(m_{0}\right)}\left(\pi a_{0} z\right) \cot ^{\left(m_{1}\right)}\left(\pi a_{1} z\right) \cot ^{\left(m_{2}\right)}\left(\pi a_{2} z\right) .
$$

By the pairwise-prime condition, the poles of $f$ are all simple with exception of the pole at $z=0$ (again we may choose our integration path such that 0 is contained inside). The residues at $z=k / a_{0}$, for example, yield, as in the proof of Theorem 2, 


$$
\begin{aligned}
& \sum_{k=1}^{a_{0}-1} \operatorname{Res}\left(f(z), z=\frac{k}{a_{0}}\right) \\
& \quad=\frac{1}{\pi}(-1)^{m_{0}} m_{0} ! \sum_{\substack{l_{1}, l_{2} \geq 0 \\
l_{1}+l_{2}=m_{0}}} \frac{a_{1}^{l_{1}} a_{2}^{l_{2}}}{l_{1} ! l_{2} !} \mathfrak{c}\left(\begin{array}{c|cc}
a_{0} & a_{1} & a_{2} \\
m_{0} & m_{1}+l_{1} & m_{2}+l_{2} \\
0 & 0 & 0
\end{array}\right) .
\end{aligned}
$$

Similar expressions are obtained for the other non-zero poles of $f$. To get the residue at $z=0$, we use the following expansion of the cotangent, which follows directly from the definition (6) of the Bernoulli polynomials [BS]:

$$
\cot z=\frac{1}{z}+\sum_{k \geq 1} \frac{(-1)^{k} 2^{2 k} B_{2 k}}{(2 k) !} z^{2 k-1} .
$$

Hence

$$
\cot ^{(m)} z=\frac{(-1)^{m} m !}{z^{m+1}}+\sum_{2 k \geq m+1} \frac{(-1)^{k} 2^{2 k-1} B_{2 k}}{k(2 k-1-m) !} z^{2 k-1-m},
$$

from which we obtain

$$
\operatorname{Res}(f(z), z=0)=-\frac{1}{\pi} \phi\left(a_{0}, a_{1}, a_{2}, m_{0}, m_{1}, m_{2}\right) .
$$

Since not all $m_{0}, m_{1}, m_{2}$ are zero, the sum of the residues of $f$ vanishes as in the proof of Theorem 2, and the statement follows.

Next we prove the reciprocity law for the cotangent sums [Di2]. Recall that

$$
\mathfrak{c}(a, b, c ; x, y, z)=\mathfrak{c}\left(\begin{array}{c|cc}
c & a & b \\
0 & 0 & 0 \\
z & x & y
\end{array}\right)
$$

It is easy to see that the reciprocity law of Meyer and Sczech [MS] is a special case of

COROllary 9 (Dieter). Let $a, b, c \in \mathbb{N}$ be pairwise relatively prime, and define $A, B, C$ by

$$
A b c+B c a+C a b=1 .
$$

Let $x, y, z \in \mathbb{R}$, not all integers, and set

$$
x^{\prime}=c y-b z, \quad y^{\prime}=a z-c x, \quad z^{\prime}=b x-a y .
$$

Finally, let

$$
\delta(x)= \begin{cases}1 & \text { if } x \in \mathbb{Z} \\ 0 & \text { else }\end{cases}
$$

Then 


$$
\begin{aligned}
\mathfrak{c}(a, b, c ; x, y, z)+\mathfrak{c}(b, c, a ; & y, z, x)+\mathfrak{c}(c, a, b ; z, x, y) \\
= & -1-\frac{c}{a b} \delta\left(z^{\prime}\right) \cot ^{(1)} \pi\left(A c x^{\prime}-(B c+C b) y^{\prime}\right) \\
& -\frac{a}{b c} \delta\left(x^{\prime}\right) \cot ^{(1)} \pi\left(B a y^{\prime}-(C a+A c) z^{\prime}\right) \\
& -\frac{b}{a c} \delta\left(y^{\prime}\right) \cot ^{(1)} \pi\left(C b z^{\prime}-(A b+B a) x^{\prime}\right) .
\end{aligned}
$$

Proof. As in the proof of Theorem 2, we use the function

$$
f(w)=\cot \pi(a w-x) \cot \pi(b w-y) \cot \pi(c w-z) .
$$

If $f$ has only simple poles, the statement is a direct special case of Theorem 2 . Otherwise, suppose we have a double pole $w_{p}$, that is, there exist integers $m$ and $n$ such that (for example)

$$
w_{p}=\frac{m+x}{a}=\frac{n+y}{b} .
$$

To compute the residue of $f$ at this pole, we use once again the Laurent series (10) and (11) of the cotangent to obtain

$$
\operatorname{Res}\left(f(w), w=w_{p}\right)=\frac{c}{\pi a b} \cot ^{(1)} \pi\left(c w_{p}-z\right)
$$

We obtain similar residues for the simple poles as before, and the residue theorem yields an identity. That this identity is equivalent to Dieter's can be easily seen by following the remarks in [Di2] just before Theorem 2.3.

The last special case is one in general "dimension", Zagier's higherdimensional Dedekind sums $[\mathrm{Za}]$. Recall that

$$
\mathfrak{s}\left(a_{0} ; a_{1}, \ldots, a_{d}\right)=(-1)^{d / 2} \mathfrak{c}\left(\begin{array}{c|ccc}
a_{0} & a_{1} & \ldots & a_{d} \\
0 & 0 & \ldots & 0 \\
0 & 0 & \ldots & 0
\end{array}\right) .
$$

COROLlary 10 (Zagier). If $a_{0}, \ldots, a_{d} \in \mathbb{N}$ are pairwise relatively prime then

$$
\sum_{n=0}^{d} \mathfrak{s}\left(a_{n} ; a_{0}, \ldots, \widehat{a_{n}}, \ldots, a_{d}\right)=1-h\left(a_{0}, \ldots, a_{d}\right)
$$

where

$$
h\left(a_{0}, \ldots, a_{d}\right):=\frac{2^{d}}{a_{0} \ldots a_{d}} \sum_{\begin{array}{c}
k_{0}, \ldots, k_{d} \geq 0 \\
k_{0}+\ldots+k_{d}=d / 2
\end{array}} \frac{B_{2 k_{0}} \ldots B_{2 k_{d}}}{\left(2 k_{0}\right) ! \ldots\left(2 k_{d}\right) !} a_{0}^{2 k_{0}} \ldots a_{d}^{2 k_{d}} .
$$

This description of $h\left(a_{0}, \ldots, a_{d}\right)$ in terms of Bernoulli numbers was first used by Berndt [Be]; however, it is easily seen to be equivalent to the version given by Zagier. It is interesting to note that $h\left(a_{0}, \ldots, a_{d}\right)$ can be expressed in terms of Hirzebruch $L$-functions [Za]. 
Proof. This time consider the function

$$
f(z)=\cot \pi a_{0} z \ldots \cot \pi a_{d} z .
$$

As before, $f$ has simple poles aside from the pole at $z=0$. The residues are calculated as usual, for example,

$$
\begin{aligned}
\sum_{k=1}^{a_{0}-1} \operatorname{Res}\left(f(z), z=\frac{k}{a_{0}}\right) & =\frac{1}{\pi a_{0}} \sum_{k=1}^{a_{0}-1} \cot \frac{\pi k a_{1}}{a_{0}} \ldots \cot \frac{\pi k a_{d}}{a_{0}} \\
& =\frac{(-1)^{d / 2}}{\pi} \mathfrak{s}\left(a_{0} ; a_{1}, \ldots, a_{d}\right) .
\end{aligned}
$$

The residue at 0 can be computed through rewriting (12) as

$$
z \cot z=\sum_{k \geq 0} \frac{(-1)^{k} 2^{2 k} B_{2 k}}{(2 k) !} z^{2 k}
$$

Hence

$$
f(z)=\frac{1}{a_{0} \ldots a_{d}(\pi z)^{d+1}} \prod_{j=0}^{d} \sum_{k_{j} \geq 0} \frac{(-1)^{k_{j}} 2^{2 k_{j}} B_{2 k_{j}}}{\left(2 k_{j}\right) !}\left(\pi a_{j} z\right)^{2 k_{j}},
$$

and we obtain the residue

$$
\begin{gathered}
\operatorname{Res}(f(z), z=0) \frac{(-1)^{d / 2} 2^{d}}{\pi a_{0} \ldots a_{d}} \sum_{\begin{array}{c}
k_{0}, \ldots, k_{d} \geq 0 \\
k_{0}+\ldots+k_{d}=d / 2
\end{array}} \frac{B_{2 k_{0}} \ldots B_{2 k_{d}}}{\left(2 k_{0}\right) ! \ldots\left(2 k_{d}\right) !} a_{0}^{2 k_{0}} \ldots a_{d}^{2 k_{d}} \\
=\frac{(-1)^{d / 2}}{\pi} h\left(a_{0}, \ldots, a_{d}\right) .
\end{gathered}
$$

It remains to apply the residue theorem and (9).

5. Petersson-Knopp identities. Knopp applied in [Kno] Hecke operators to $\log \eta$ to arrive at Theorem 3. This identity was stated by Petersson in the 1970's with additional congruence restrictions on $a$ and $b$. For $n$ prime, the Petersson-Knopp identity was already known to Dedekind [De]. Theorem 3 was generalized by Parson and Rosen [PR] to Dedekind-Bernoulli sums, by Apostol and $\mathrm{Vu}[\mathrm{AV}]$ to their "sums of Dedekind type", and, most broadly, by Zheng [Zh] to what we will call sums of Dedekind type with weight $\left(m_{1}, m_{2}\right)$. We will state and further generalize Zheng's PeterssonKnopp identity after the following

Definition 2. Let $a, a_{1}, \ldots, a_{d} \in \mathbb{N}$. The sum

$$
S\left(a ; a_{1}, \ldots, a_{d}\right):=\sum_{k \bmod a} f_{1}\left(\frac{k a_{1}}{a}\right) \ldots f_{d}\left(\frac{k a_{d}}{a}\right)
$$


is said to be of Dedekind type with weight $\left(m_{1}, \ldots, m_{d}\right)$ if for all $j=1, \ldots, d$, $f_{j}(x+1)=f_{j}(x)$, and for all $a \in \mathbb{N}$,

$$
\sum_{k \bmod a} f_{j}\left(x+\frac{k}{a}\right)=a^{m_{j}} f_{j}(a x) .
$$

Note that the Bernoulli functions $\bar{B}_{m}(x)$ satisfy (13) (with "weight" $-m+1$ ), as do the functions $\cot ^{(m)}(\pi x)$ (with "weight" $m+1$ ). Zheng's theorem is the "two-dimensional" $(d=2)$ case of the following

TheOREm 11. Let $n, a, a_{1}, \ldots, a_{d} \in \mathbb{N}$. If

$$
S\left(a ; a_{1}, \ldots, a_{d}\right):=\sum_{k \bmod a} f_{1}\left(\frac{k a_{1}}{a}\right) \ldots f_{d}\left(\frac{k a_{d}}{a}\right)
$$

is of Dedekind type with weight $\left(m_{1}, \ldots, m_{d}\right)$ then

$$
\begin{aligned}
\sum_{b \mid n} b^{-m_{1}-\ldots-m_{d}} \sum_{r_{1}, \ldots, r_{d} \bmod b} S\left(a b ; \frac{n}{b} a_{1}+r_{1} a, \ldots, \frac{n}{b} a_{d}+r_{d} a\right) \\
=n \sigma_{d-1-m_{1}-\ldots-m_{d}}(n) S\left(a ; a_{1}, \ldots, a_{d}\right) .
\end{aligned}
$$

Our proof is a relatively straightforward extension of Zheng's [Zh] proof for $d=2$. We need the following two identities:

LEMMA 12. Let $a, a_{1}, \ldots, a_{d} \in \mathbb{N}$. If

$$
S\left(a ; a_{1}, \ldots, a_{d}\right):=\sum_{k \bmod a} f_{1}\left(\frac{k a_{1}}{a}\right) \ldots f_{d}\left(\frac{k a_{d}}{a}\right)
$$

is of Dedekind type with weight $\left(m_{1}, \ldots, m_{d}\right)$ then for all $j=1, \ldots, d$,

$$
\sum_{k \bmod a} f_{j}\left(x+\frac{k b}{a}\right)=(a, b)^{1-m_{j}} a^{m_{j}} f_{j}\left(\frac{a x}{(a, b)}\right)
$$

and

$$
S\left(a b ; a_{1} b, \ldots, a_{d} b\right)=b S\left(a ; a_{1}, \ldots, a_{d}\right) .
$$

Proof. For (14), note first that if $(a, b)=1$, the statement is vacuous. Next, if $a$ and $b$ are not relatively prime, let $a^{\prime}=a /(a, b)$ and $b^{\prime}=b /(a, b)$. Then

$$
\begin{aligned}
\sum_{k \bmod a} f_{j}\left(x+\frac{k b}{a}\right) & =\sum_{k \bmod a} f_{j}\left(x+\frac{k b^{\prime}}{a^{\prime}}\right)=(a, b) \sum_{k \bmod a^{\prime}} f_{j}\left(x+\frac{k b^{\prime}}{a^{\prime}}\right) \\
& =(a, b) \sum_{k \bmod a^{\prime}} f_{j}\left(x+\frac{k}{a^{\prime}}\right) \\
& =(a, b) a^{\prime m_{j}} f_{j}\left(a^{\prime} x\right)=(a, b)^{1-m_{j}} a^{m} f_{j}\left(\frac{a x}{(a, b)}\right) .
\end{aligned}
$$


For (15), we have

$$
\begin{aligned}
S\left(a b ; a_{1} b, \ldots, a_{d} b\right) & =\sum_{k \bmod a b} f_{1}\left(\frac{k a_{1}}{a}\right) \ldots f_{d}\left(\frac{k a_{d}}{a}\right) \\
& =b \sum_{k \bmod a} f_{1}\left(\frac{k a_{1}}{a}\right) \ldots f_{d}\left(\frac{k a_{d}}{a}\right)=b S\left(a ; a_{1}, \ldots, a_{d}\right) .
\end{aligned}
$$

Proof of Theorem 11. We will make use of two properties of the Möbius $\mu$-function

$$
\mu(n)= \begin{cases}1 & \text { if } n=1 \\ (-1)^{m} & \text { if } n=p_{1} \ldots p_{m} \text { is square-free } \\ 0 & \text { otherwise }\end{cases}
$$

namely,

$$
\sum_{d \mid n} \mu(d)= \begin{cases}1 & \text { if } n=1 \\ 0 & \text { otherwise }\end{cases}
$$

and

$$
\sum_{\substack{k=1 \\(k, b)=1}}^{a b} f(k)=\sum_{t \mid b} \mu(t) \sum_{k=1}^{a b / t} f(t k)
$$

These suffice to prove our statement:

$$
\begin{aligned}
\sum_{b \mid n} b^{-} & m_{1}-\ldots-m_{d} \sum_{r_{1}, \ldots, r_{d} \bmod b} S\left(a b ; \frac{n a_{1}}{b}+r_{1} a, \ldots, \frac{n a_{d}}{b}+r_{d} a\right) \\
= & \sum_{b \mid n} b^{-m_{1}-\ldots-m_{d}} \sum_{\substack{r_{1}, \ldots, r_{d} \bmod b \\
k \bmod a b}} f_{1}\left(\frac{k n a_{1}}{a b^{2}}+\frac{k r_{1}}{b}\right) \ldots f_{d}\left(\frac{k n a_{d}}{a b^{2}}+\frac{k r_{d}}{b}\right) \\
\stackrel{(14)}{=} & \sum_{b \mid n} b^{-m_{1}-\ldots-m_{d}} \sum_{k \bmod a b}(k, b)^{1-m_{1}} b^{m_{1}} \\
& \times f_{1}\left(\frac{k n a_{1}}{a b(a, b)}\right) \ldots(k, b)^{1-m_{d}} b^{m_{d}} f_{d}\left(\frac{k n a_{d}}{a b(a, b)}\right) \\
= & \sum_{b \mid n} \sum_{k \bmod a b}(k, b)^{d-m_{1}-\ldots-m_{d}} f_{1}\left(\frac{k n a_{1}}{a b(a, b)}\right) \ldots f_{d}\left(\frac{k n a_{d}}{a b(a, b)}\right) \\
= & \sum_{b \mid n} \sum_{c \mid b} c^{d-m_{1}-\ldots-m_{d}} \sum_{\substack{k \bmod a b \\
(k, b)=c}} f_{1}\left(\frac{k n a_{1}}{a b c}\right) \ldots f_{d}\left(\frac{k n a_{d}}{a b c}\right)
\end{aligned}
$$




$$
\begin{aligned}
& =\sum_{b \mid n} \sum_{c \mid b} c^{d-m_{1}-\ldots-m_{d}} \sum_{\substack{k \bmod a b / c \\
(k, b / c)=1}} f_{1}\left(\frac{k n a_{1}}{a b}\right) \ldots f_{d}\left(\frac{k n a_{d}}{a b}\right) \\
& \stackrel{(17)}{=} \sum_{b \mid n} \sum_{c \mid b} c^{d-m_{1}-\ldots-m_{d}} \sum_{t \mid b / c} \mu(t) \sum_{k \bmod a b /(c t)} f_{1}\left(\frac{t k n a_{1}}{a b}\right) \ldots f_{d}\left(\frac{t k n a_{d}}{a b}\right) \\
& =\sum_{b \mid n} \sum_{c \mid b} c^{d-m_{1}-\ldots-m_{d}} \sum_{t \mid b / c} \mu(t) S\left(\frac{a b}{c t} ; \frac{n a_{1}}{c}, \ldots, \frac{n a_{d}}{c}\right) \\
& =\sum_{c t e \mid n} c^{d-m_{1}-\ldots-m_{d}} \mu(t) S\left(a e ; \frac{n a_{1}}{c}, \ldots, \frac{n a_{d}}{c}\right) \\
& =\sum_{c e \mid n} c^{d-m_{1}-\ldots-m_{d}} S\left(a e ; \frac{n a_{1}}{c}, \ldots, \frac{n a_{d}}{c}\right) \sum_{t \mid n /(c e)} \mu(t) \\
& \stackrel{(16)}{=} \sum_{c e=n} c^{d-m_{1}-\ldots-m_{d}} S\left(a e ; a_{1} e, \ldots, a_{d} e\right) \\
& \stackrel{(15)}{=} \sigma_{d-1-m_{1}-\ldots-m_{d}}(n) S\left(a ; a_{1}, \ldots, a_{d}\right) . \text { - }
\end{aligned}
$$

The fact that the Dedekind cotangent sums

$$
a_{0}^{m_{0}+1} \mathfrak{c}\left(\begin{array}{c|ccc}
a_{0} & a_{1} & \ldots & a_{d} \\
m_{0} & m_{1} & \ldots & m_{d} \\
0 & 0 & \ldots & 0
\end{array}\right)=\sum_{k \bmod a_{0}} \prod_{j=1}^{d} \cot ^{\left(m_{j}\right)} \frac{\pi k a_{j}}{a_{0}}
$$

have weight $\left(m_{1}+1, \ldots, m_{d}+1\right)$ immediately yields the Petersson-Knopplike Theorem 4.

A particularly simple form of Theorem 4 is achieved for Zagier's higherdimensional Dedekind sums (the case $m_{0}=\ldots=m_{d}=0$ ): with

$$
n \sigma_{-1}(n)=\sum_{d \mid n} \frac{n}{d}=\sum_{d \mid n} d=\sigma(n),
$$

we obtain

Corollary 13. For $n, a_{0}, \ldots, a_{d} \in \mathbb{N}$,

$$
\begin{aligned}
\sum_{b \mid n} b^{1-d} \sum_{r_{1}, \ldots, r_{d} \bmod b} \mathfrak{s}\left(a_{0} b ; \frac{n}{b} a_{1}+r_{1} a_{0}, \ldots,\right. & \left.\frac{n}{b} a_{d}+r_{d} a_{0}\right) \\
& =\sigma(n) \mathfrak{s}\left(a_{0} ; a_{1}, \ldots, a_{d}\right) .
\end{aligned}
$$


6. Proof of the polynomial-time computability. In this last section, we prove that the Dedekind cotangent sums are computable in polynomial time (Theorem 5). Our proof is similar to the last section of [BR], where the computability of Zagier's higher-dimensional Dedekind sums was shown. Again we will "merge" two theorems in combinatorial geometry, due to Barvinok [Ba] and Diaz-Robins [DR]. The latter allows us to express generating functions for the lattice point count in cones in terms of cotangents; Barvinok's theorem tells us that the respective rational functions are computable. The usual generating function for lattice point enumeration for a $d$-dimensional cone $\mathcal{K}$,

$$
F_{\mathcal{K}}(\mathbf{q})=F_{\mathcal{K}}\left(q_{1}, \ldots, q_{d}\right):=\sum_{\mathbf{m}=\left(m_{1}, \ldots, m_{d}\right) \in \mathcal{K} \cap \mathbb{Z}^{d}} q_{1}^{m_{1}} \ldots q_{d}^{m_{d}},
$$

assumes in $[\mathrm{DR}]$ an exponential variable:

Theorem 14 (Diaz-Robins). Suppose the cone $\mathcal{K} \subset \mathbb{R}^{d}$ is generated by the positive real span of the integer vectors $\mathbf{v}_{1}, \ldots, \mathbf{v}_{d} \in \mathbb{Z}^{d}$ such that the $d \times d$-matrix $M=\left(a_{i j}\right)$, whose column vectors are $\mathbf{v}_{1}, \ldots, \mathbf{v}_{d}$, is lowertriangular. Let $p_{k}:=a_{11} \ldots a_{k k}(k=1, \ldots, d)$ and $G:=\left(\mathbb{Z} / p_{1} \mathbb{Z}\right) \times \ldots \times$ $\left(\mathbb{Z} / p_{d} \mathbb{Z}\right)$. Then

$$
\sum_{\mathbf{m} \in \mathcal{K} \cap \mathbb{Z}^{d}} e^{-2 \pi\langle\mathbf{m}, \mathbf{s}\rangle}=\frac{1}{2^{d}|G|} \sum_{\mathbf{r} \in G} \prod_{k=1}^{d}\left(1+\operatorname{coth} \frac{\pi}{p_{k}}\left\langle\mathbf{s}+i \mathbf{r}, \mathbf{v}_{k}\right\rangle\right) .
$$

Here $\langle$,$\rangle denotes the usual scalar product in \mathbb{R}^{d}$.

We note that the assumption on $M$ being lower-triangular is not crucial for practical purposes: by Hermite normal form, any cone generated by integer vectors is unimodular equivalent to a cone described by a lowertriangular matrix.

TheOREM 15 (Barvinok). For fixed dimension $d$, the rational function $F_{\mathcal{K}}(\mathbf{q})$ is polynomial-time computable in the input size of $\mathcal{K}$.

These two powerful theorems combined allow us to compute the Dedekind cotangent sum in polynomial time:

Proof of Theorem 5. It suffices to prove the polynomial-time computability of

$$
\sum_{k \bmod } \prod_{j=1}^{d} \cot \pi\left(\frac{k a_{j}}{a}+z_{j}\right)
$$

We will do this inductively by constructing a cone whose generating function has the above Dedekind cotangent sum + "lower-dimensional" sums, that is, Dedekind cotangent sums with a lower number of factors. More precisely, 
we will construct a cone whose generating function will be

$$
\sum_{k \bmod a}\left(1+\cot \pi\left(\frac{k a_{1}}{a}+z_{1}\right)\right) \ldots\left(1+\cot \pi\left(\frac{k a_{d}}{a}+z_{d}\right)\right) .
$$

By induction and Barvinok's Theorem 15, this will prove the computability of the Dedekind cotangent sum. Let $\mathcal{K}$ be the positive real span of

$$
\left(\begin{array}{c}
a \\
0 \\
\vdots \\
0 \\
b_{1}
\end{array}\right),\left(\begin{array}{c}
0 \\
a \\
0 \\
\vdots \\
0 \\
b_{2}
\end{array}\right), \ldots,\left(\begin{array}{c}
0 \\
\vdots \\
0 \\
a \\
b_{d-1}
\end{array}\right),\left(\begin{array}{c}
0 \\
\\
\vdots \\
0 \\
a
\end{array}\right) .
$$

The integers $b_{1}, \ldots, b_{d-1}$ are to be chosen later. We will repeatedly use the fact that

$$
\sum_{k \bmod a} \operatorname{coth} \pi\left(\frac{i k}{a}+z\right)=a \operatorname{coth} \pi a z
$$

To apply Theorem 14 to $\mathcal{K}$, note that in our case $p_{k}=a^{k}$. Hence

$$
\begin{aligned}
& \sum_{\mathbf{m} \in \mathcal{K} \cap \mathbb{Z}^{d}} e^{-2 \pi\langle\mathbf{m}, \mathbf{s}\rangle}=\frac{1}{2^{d} a^{d(d+1) / 2}} \sum_{r_{j} \bmod a^{j}} \prod_{k=1}^{d}\left(1+\operatorname{coth} \frac{\pi}{a^{k}}\left\langle\mathbf{s}+i \mathbf{r}, \mathbf{v}_{k}\right\rangle\right) \\
& =\frac{1}{2^{d} a^{d(d+1) / 2}} \sum_{\substack{r_{j} \bmod a^{j} \\
(j=1, \ldots, d)}}\left(1+\operatorname{coth} \frac{\pi}{a}\left(\left(s_{1}+i r_{1}\right) a+\left(s_{d}+i r_{d}\right) b_{1}\right)\right) \\
& \times\left(1+\operatorname{coth} \frac{\pi}{a^{2}}\left(\left(s_{2}+i r_{2}\right) a+\left(s_{d}+i r_{d}\right) b_{2}\right)\right) \\
& \times \ldots \times\left(1+\operatorname{coth} \frac{\pi}{a^{d}}\left(s_{d}+i r_{d}\right) a\right) \\
& =\frac{1}{2^{d} a^{d(d+1) / 2-1}} \sum_{\substack{r_{j} \bmod a^{j} \\
(j=3, \ldots, d)}}\left(1+\operatorname{coth} \pi\left(s_{1}+\left(s_{d}+i r_{d}\right) \frac{b_{1}}{a}\right)\right) \\
& \times\left(1+\operatorname{coth} \frac{\pi}{a^{3}}\left(\left(s_{3}+i r_{3}\right) a+\left(s_{d}+i r_{d}\right) b_{3}\right)\right) \\
& \times \ldots \times\left(1+\operatorname{coth} \frac{\pi}{a^{d-1}}\left(s_{d}+i r_{d}\right)\right) \\
& \times \sum_{r_{2} \bmod a^{2}}\left(1+\operatorname{coth} \pi\left(\frac{i r_{2}}{a}+\frac{s_{2}}{a}+\left(s_{d}+i r_{d}\right) \frac{b_{2}}{a^{2}}\right)\right)
\end{aligned}
$$




$$
\begin{aligned}
& \stackrel{(18)}{=} \frac{1}{2^{d} a^{d(d+1) / 2-3}} \sum_{\substack{r_{j} \bmod a^{j} \\
(j=4, \ldots, d)}}\left(1+\operatorname{coth} \pi\left(s_{1}+\left(s_{d}+i r_{d}\right) \frac{b_{1}}{a}\right)\right) \\
& \times\left(1+\operatorname{coth} \pi\left(s_{2}+\left(s_{d}+i r_{d}\right) \frac{b_{2}}{a}\right)\right) \\
& \times\left(1+\operatorname{coth} \frac{\pi}{a^{4}}\left(\left(s_{4}+i r_{4}\right) a+\left(s_{d}+i r_{d}\right) b_{4}\right)\right) \\
& \times \ldots \times\left(1+\operatorname{coth} \frac{\pi}{a^{d-1}}\left(s_{d}+i r_{d}\right)\right) \\
& \times \sum_{r_{3} \bmod a^{3}}\left(1+\operatorname{coth} \pi\left(\frac{i r_{3}}{a^{2}}+\frac{s_{3}}{a^{2}}+\left(s_{d}+i r_{d}\right) \frac{b_{3}}{a^{3}}\right)\right) \\
& \stackrel{(18)}{=} \ldots=\frac{1}{2^{d} a^{d}} \sum_{r_{d} \bmod a^{d}}\left(1+\operatorname{coth} \pi\left(s_{1}+\left(s_{d}+i r_{d}\right) \frac{b_{1}}{a}\right)\right) \times \ldots \\
& \times\left(1+\operatorname{coth} \pi\left(s_{d-1}+\left(s_{d}+i r_{d}\right) \frac{b_{d-1}}{a}\right)\right)\left(1+\operatorname{coth} \frac{\pi}{a^{d-1}}\left(s_{d}+i r_{d}\right)\right) \\
& =\frac{1}{2^{d} a^{d}} \sum_{n=1}^{a^{d-1}} \sum_{k=1}^{a}\left(1+\operatorname{coth} \pi\left(s_{1}+\left(s_{d}+i(n a+k)\right) \frac{b_{1}}{a}\right)\right) \\
& \times \ldots \times\left(1+\operatorname{coth} \pi\left(s_{d-1}+\left(s_{d}+i(n a+k)\right) \frac{b_{d-1}}{a}\right)\right) \\
& \times\left(1+\operatorname{coth} \frac{\pi}{a^{d-1}}\left(s_{d}+i(n a+k)\right)\right) \\
& =\frac{1}{2^{d} a^{d-1}} \sum_{k=1}^{a}\left(1+\operatorname{coth} \pi\left(s_{1}+\left(s_{d}+i k\right) \frac{b_{1}}{a}\right)\right) \\
& \times \ldots \times\left(1+\operatorname{coth} \pi\left(s_{d-1}+\left(s_{d}+i k\right) \frac{b_{d-1}}{a}\right)\right) \\
& \times \sum_{n=1}^{a^{d-2}}\left(1+\operatorname{coth} \pi\left(\frac{i n}{a^{d-2}}+\frac{s_{d}}{a^{d-1}}+\frac{i k}{a^{d-1}}\right)\right) \\
& \stackrel{(18)}{=} \frac{1}{2^{d} a} \sum_{k=1}^{a}\left(1+\operatorname{coth} \pi\left(s_{1}+\left(s_{d}+i k\right) \frac{b_{1}}{a}\right)\right) \times \ldots \\
& \times\left(1+\operatorname{coth} \pi\left(s_{d-1}+\left(s_{d}+i k\right) \frac{b_{d-1}}{a}\right)\right)\left(1+\operatorname{coth} \pi\left(\frac{s_{d}}{a}+\frac{i k}{a}\right)\right) .
\end{aligned}
$$

If we now choose $b_{j}=a_{d}^{-1} a_{j}(j=1, \ldots, d-1)$, where $a_{d}^{-1} a_{d} \equiv 1 \bmod a$, and

$$
s_{j}=i z_{j}-i z_{d} b_{j} \quad(j=1, \ldots, d-1), \quad s_{d}=i a z_{d},
$$

this generating function becomes 
$\sum_{\mathbf{m} \in \mathcal{K} \cap \mathbb{Z}^{d}} e^{-2 \pi\langle\mathbf{m}, \mathbf{s}\rangle}$

$$
=\frac{1}{2^{d} a} \sum_{k \bmod a}\left(1+\cot \pi\left(\frac{k a_{1}}{a}+z_{1}\right)\right) \ldots\left(1+\cot \pi\left(\frac{k a_{d}}{a}+z_{d}\right)\right) .
$$

7. Closing remarks. There remain many open questions. First, for all Dedekind sums there are closed formulas for special cases, for example, $\mathfrak{s}(1, a)$. Many of such formulas can be found in [BY]. The Dedekind cotangent sum and its variations attain such closed formulas for special variables; it is not clear how far these cases lead. Second, it seems desirable to have a parallel theory for the respective Dedekind sum in which the cotangents get replaced by Bernoulli functions. This will most certainly require different methods than the ones used in this paper. Finally, we can apply our very general Petersson-Knopp-like Theorem 11 to various other sums, for example, to such "Dedekind Bernoulli sums".

Acknowledgements. I am grateful to Sinai Robins for good discussions and Thomas Zaslavsky for many helpful comments on a previous version of this paper.

\section{References}

[Al] G. Almkvist, Asymptotic formulas and generalized Dedekind sums, Experiment. Math. 7 (1998), 343-359.

[Ap] T. M. Apostol, Generalized Dedekind sums and transformation formulae of certain Lambert series, Duke Math. J. 17 (1950), 147-157.

[AV] T. M. Apostol and T. H. Vu, Identities for sums of Dedekind type, J. Number Theory 14 (1982), 391-396.

[Ba] A. I. Barvinok, Computing the Ehrhart polynomial of a convex lattice polytope, Discrete Comput. Geom. 12 (1994), 35-48.

[BR] M. Beck and S. Robins, Dedekind sums: a combinatorial-geometric viewpoint, Discrete Math. Theoret. Comput. Sci., to appear.

[Be] B. C. Berndt, Reciprocity theorems for Dedekind sums and generalizations, Adv. Math. 23 (1977), 285-316.

[BD] B. C. Berndt and U. Dieter, Sums involving the greatest integer function and Riemann-Stieltjes integration, J. Reine Angew. Math. 337 (1982), 208-220.

[BY] B. C. Berndt and B. P. Yeap, Explicit evaluations and reciprocity theorems for finite trigonometric sums, Adv. Appl. Math. 29 (2002), 358-385.

[BV] M. Brion and M. Vergne, Lattice points in simple polytopes, J. Amer. Math. Soc. 10 (1997), 371-392.

[BS] I. N. Bronstein und K. A. Semendjajew, Taschenbuch der Mathematik, Harri Deutsch, Thun, 1989.

[C1] L. Carlitz, Some theorems on generalized Dedekind sums, Pacific J. Math. 3 (1953), 513-522.

[C2] -, A note on generalized Dedekind sums, Duke Math. J. 21 (1954), 399-404. 
[De] R. Dedekind, Erläuterungen zu den Fragmenten XXVIII, in: Collected Works of Bernhard Riemann, Dover, New York, 1953, 466-478.

[DR] R. Diaz and S. Robins, The Ehrhart polynomial of a lattice polytope, Ann. of Math. 145 (1997), 503-518.

[Di1] U. Dieter, Das Verhalten der Kleinschen Funktionen $\log \sigma_{g, h}\left(w_{1}, w_{2}\right)$ gegenüber Modultransformationen und verallgemeinerte Dedekindsche Summen, J. Reine Angew. Math. 201 (1959), 37-70.

[Di2] -, Cotangent sums, a further generalization of Dedekind sums, J. Number Theory 18 (1984), 289-305.

[H] U. Halbritter, Some new reciprocity formulas for generalized Dedekind sums, Resultate Math. 8 (1985), 21-46.

[HWZ] R. R. Hall, J. C. Wilson and D. Zagier, Reciprocity formulae for general Dedekind-Rademacher sums, Acta Arith. 73 (1995), 389-396.

[HZ] F. Hirzebruch and D. Zagier, The Atiyah-Singer Theorem and Elementary Number Theory, Publish or Perish, Boston, 1974.

[Kno] M. I. Knopp, Hecke operators and an identity for Dedekind sums, J. Number Theory 12 (1980), 2-9.

[Knu] D. Knuth, The Art of Computer Programming, Vol. 2, Addison-Wesley, Reading, MA, 1981.

[Me] C. Meyer, Über einige Anwendungen Dedekindscher Summen, J. Reine Angew. Math. 198 (1957), 143-203.

[MS] W. Meyer und R. Sczech, Über eine topologische und zahlentheoretische Anwendung von Hirzebruchs Spitzenauflösung, Math. Ann. 240 (1979), 69-96.

[Mi] M. Mikolás, On certain sums generating the Dedekind sums and their reciprocity laws, Pacific J. Math. 7 (1957), 1167-1178.

[Mo] L. J. Mordell, Lattice points in a tetrahedron and generalized Dedekind sums, J. Indian Math. Soc. 15 (1951), 41-46.

[PR] L. A. Parson and K. Rosen, Hecke operators and Lambert series, Math. Scand. 49 (1981), 5-14.

[P] J. Pommersheim, Toric varieties, lattice points, and Dedekind sums, Math. Ann. 295 (1993), 1-24.

[R] H. Rademacher, Some remarks on certain generalized Dedekind sums, Acta Arith. 9 (1964), 97-105.

[RG] H. Rademacher and E. Grosswald, Dedekind Sums, Carus Math. Monogr. 16, Math. Assoc. America, Washington, DC, 1972.

[S] D. Solomon, Algebraic properties of Shintani's generating functions: Dedekind sums and cocycles on $\mathrm{PGL}_{2}(\mathbb{Q})$, Compositio Math. 112 (1998), 333-362.

[T] L. Takács, On generalized Dedekind sums, J. Number Theory 11 (1979), 264-272.

[Za] D. Zagier, Higher dimensional Dedekind sums, Math. Ann. 202 (1973), 149-172.

[Zh] Z. Zheng, The Petersson-Knopp identity for the homogeneous Dedekind sums, J. Number Theory 57 (1996), 223-230.

Department of Mathematical Sciences

State University of New York

Binghamton, NY 13902-6000, U.S.A.

E-mail: matthias@math.binghamton.edu

http://www.math.binghamton.edu/matthias 